\title{
Algebraic cobordism of bundles on varieties
}

Received February 21, 2010, and in revised form January 12, 2011

\begin{abstract}
The double point relation defines a natural theory of algebraic cobordism for bundles on varieties. We construct a simple basis (over $\mathbb{Q}$ ) of the corresponding cobordism groups over $\operatorname{Spec}(\mathbb{C})$ for all dimensions of varieties and ranks of bundles. The basis consists of split bundles over products of projective spaces. Moreover, we prove that the full theory for bundles on varieties is an extension of scalars of standard algebraic cobordism.
\end{abstract}

\section{Introduction}

\subsection{Algebraic cobordism}

A successful theory of algebraic cobordism has been constructed in [6] from Quillen's axiomatic perspective. The result $\Omega_{*}$ is the universal oriented Borel-Moore homology theory of schemes, yielding the universal oriented Borel-Moore cohomology theory $\Omega^{*}$ for the subcategory of smooth schemes.

Let $k$ be a field of characteristic 0 . Let $\mathbf{S c h}_{k}$ be the category of separated schemes of finite type over $k$, and let $\mathbf{S m}_{k}$ be the full subcategory of smooth quasi-projective $k$ schemes. A geometric presentation of algebraic cobordism in characteristic 0 via double point relations is given in [7].

\subsection{Double point degenerations}

Let $Y \in \mathbf{S m}_{k}$ be of pure dimension. A morphism $\pi: Y \rightarrow \mathbb{P}^{1}$ is a double point degeneration over $0 \in \mathbb{P}^{1}$ if $\pi^{-1}(0)$ can be written as $\pi^{-1}(0)=A \cup B$ where $A$ and $B$ are smooth codimension one closed subschemes of $Y$, intersecting transversely. The intersection $D=A \cap B$ is the double point locus of $\pi$ over $0 \in \mathbb{P}^{1}$. We do not require $A, B$, or $D$ to be connected. Moreover, $A, B$, and $D$ are allowed to be empty.

Let $N_{A / D}$ and $N_{B / D}$ denote the normal bundles of $D$ in $A$ and $B$ respectively. Since $O_{D}(A+B)$ is trivial,

$$
N_{A / D} \otimes N_{B / D} \cong O_{D}
$$

Y.-P. Lee: Department of Mathematics, University of Utah, Salt Lake City, UT 84112, USA; e-mail: yplee@math.utah.edu

R. Pandharipande: Department of Mathematics, Princeton University, Princeton, NJ 08544, USA; e-mail: rahulp@math.princeton.edu 
Since $O_{D} \oplus N_{A / D} \cong N_{A / D} \otimes\left(O_{D} \oplus N_{B / D}\right)$, the projective bundles

$$
\mathbb{P}\left(O_{D} \oplus N_{A / D}\right) \rightarrow D \quad \text { and } \quad \mathbb{P}\left(O_{D} \oplus N_{B / D}\right) \rightarrow D
$$

are isomorphic. Let $\mathbb{P}(\pi) \rightarrow D$ denote either of $(0.1)$.

\section{3. $\mathcal{M}(X)^{+}$}

For $X \in \mathbf{S c h}_{k}$, let $\mathcal{M}(X)$ denote the set of isomorphism classes over $X$ of projective morphisms

$$
f: Y \rightarrow X
$$

with $Y \in \mathbf{S m}_{k}$. The set $\mathcal{M}(X)$ is a monoid under disjoint union of domains and is graded by the dimension of $Y$ over $k$. Let $\mathcal{M}_{*}(X)^{+}$denote the graded group completion of $\mathcal{M}(X)$.

Alternatively, $\mathcal{M}_{n}(X)^{+}$is the free abelian group generated by morphisms $(0.2)$ where $Y$ is irreducible and of dimension $n$ over $k$. Let $[f: Y \rightarrow X] \in \mathcal{M}_{*}(X)^{+}$denote the element determined by the morphism.

\subsection{Double point relations}

Let $X \in \mathbf{S c h}_{k}$, and let $p_{1}$ and $p_{2}$ denote the projections to the first and second factors of $X \times \mathbb{P}^{1}$ respectively.

Let $Y \in \mathbf{S m}_{k}$ be of pure dimension. Let $g: Y \rightarrow X \times \mathbb{P}^{1}$ be a projective morphism for which the composition

$$
\pi=p_{2} \circ g: Y \rightarrow \mathbb{P}^{1}
$$

is a double point degeneration over $0 \in \mathbb{P}^{1}$. Let

$$
[A \rightarrow X],[B \rightarrow X],[\mathbb{P}(\pi) \rightarrow X] \in \mathcal{M}(X)^{+}
$$

be obtained from the fiber $\pi^{-1}(0)$ and the morphism $p_{1} \circ g$.

Definition 1. Let $\zeta \in \mathbb{P}^{1}(k)$ be a regular value of $\pi$. We call the map $g$ a double point cobordism with degenerate fiber over 0 and smooth fiber over $\zeta$. The associated double point relation over $X$ is

$$
\left[Y_{\zeta} \rightarrow X\right]-[A \rightarrow X]-[B \rightarrow X]+[\mathbb{P}(\pi) \rightarrow X]
$$

where $Y_{\zeta}=\pi^{-1}(\zeta)$

The relation (0.4) depends not only on the morphism $g$ and the point $\zeta$, but also on the choice of decomposition of the fiber $\pi^{-1}(0)=A \cup B$. We view (0.4) as an analog of the classical relation of rational equivalence of algebraic cycles.

Let $\mathcal{R}_{*}(X) \subset \mathcal{M}_{*}(X)^{+}$be the subgroup generated by all double point relations over $X$. Since (0.4) is a homogeneous element of $\mathcal{M}_{*}(X)^{+}, \mathcal{R}_{*}(X)$ is a graded subgroup of $\mathcal{M}_{*}(X)^{+}$. 
Definition 2. For $X \in \mathbf{S c h}_{k}$, double point cobordism $\omega_{*}(X)$ is defined by the quotient

$$
\omega_{*}(X)=\mathcal{M}_{*}(X)^{+} / \mathcal{R}_{*}(X) .
$$

A central result of [7] is the isomorphism

$$
\Omega_{*} \cong \omega_{*}
$$

which provides a geometric presentation of algebraic cobordism. Since resolution of singularities and Bertini's results are used, the isomorphism is established only when $k$ has characteristic 0 .

\subsection{Over a point}

We write $\Omega_{*}(k)$ and $\omega_{*}(k)$ for $\Omega_{*}(\operatorname{Spec}(k))$ and $\omega_{*}(\operatorname{Spec}(k))$ respectively. Let $\mathbb{L}_{*}$ be the Lazard ring [4]. The canonical map

$$
\mathbb{L}_{*} \rightarrow \Omega_{*}(k)
$$

classifying the formal group law for $\Omega_{*}$ is proven to be an isomorphism in [6, Theorem 4.3.7]. By Quillen's result for complex cobordism (in topology),

$$
\mathbb{L}_{n} \cong M U^{-2 n}(\mathrm{pt})
$$

and the well-known generators of $M U^{*}(\mathrm{pt})_{\mathbb{Q}}\left[11\right.$, Chapter VII], we see $\Omega_{*}(k) \otimes_{\mathbb{Z}} \mathbb{Q}$ is generated as a $\mathbb{Q}$-algebra by the classes of projective spaces. Then it is a consequence of (0.6) that

$$
\omega_{*}(k) \otimes_{\mathbb{Z}} \mathbb{Q}=\bigoplus_{\lambda} \mathbb{Q}\left[\mathbb{P}^{\lambda_{1}} \times \cdots \times \mathbb{P}^{\lambda_{\ell(\lambda)}}\right],
$$

where the sum is over all partitions $\lambda$. The partition $\lambda=\emptyset$ corresponds to $\left[\mathbb{P}^{0}\right]$ in grade 0 .

\subsection{Bundles}

For $X \in \mathbf{S c h}_{k}$, let $\mathcal{M}_{n, r}(X)$ denote the set of isomorphism classes over $X$ of pairs [ $f: Y \rightarrow X, E$ ] with $Y \in \mathbf{S m}_{k}$ of dimension $n, f$ projective, and $E$ a rank $r$ vector bundle on $Y$. The set $\mathcal{M}_{n, r}(X)$ is a monoid under disjoint union of domains. Let $\mathcal{M}_{n, r}(X)^{+}$ denote the group completion of $\mathcal{M}_{n, r}(X)$.

Double point relations are easily defined in the setting of pairs following [7, Section 13]. Let $Y \in \mathbf{S m}_{k}$ be of pure dimension $n+1$. Let $g: Y \rightarrow X \times \mathbb{P}^{1}$ be a projective morphism for which the composition $\pi=p_{2} \circ g: Y \rightarrow \mathbb{P}^{1}$ is a double point degeneration over $0 \in \mathbb{P}^{1}$. Let $E$ be a rank $r$ vector bundle on $Y$. Let

$$
\left[A \rightarrow X, E_{A}\right],\left[B \rightarrow X, E_{B}\right],\left[\mathbb{P}(\pi) \rightarrow X, E_{\mathbb{P}(\pi)}\right] \in \mathcal{M}_{n, r}(X)^{+}
$$

be obtained from the fiber $\pi^{-1}(0)$ and the morphism $p_{1} \circ g$. Here, $E_{A}$ and $E_{B}$ denote the restrictions of $E$ to $A$ and $B$ respectively. The restriction $E_{\mathbb{P}(\pi)}$ is defined by pull-back from $Y$ via $\mathbb{P}(\pi) \rightarrow D \subset Y$. 
Definition 3. Let $\zeta \in \mathbb{P}^{1}(k)$ be a regular value of $\pi$. The associated double point relation over $X$ is

$$
\left[Y_{\zeta} \rightarrow X, E_{Y_{\zeta}}\right]-\left[A \rightarrow X, E_{A}\right]-\left[B \rightarrow X, E_{B}\right]+\left[\mathbb{P}(\pi) \rightarrow X, E_{\mathbb{P}(\pi)}\right]
$$

where $Y_{\zeta}=\pi^{-1}(\zeta)$.

For $X \in \mathbf{S c h}_{k}$, let $\mathcal{R}_{n, r}(X) \subset M_{n, r}(X)^{+}$be the subgroup generated by all double point relations. Double point cobordism theory for bundles on varieties is defined by

$$
\omega_{n, r}(X)=\mathcal{M}_{n, r}(X)^{+} / \mathcal{R}_{n, r}(X) .
$$

The sum

$$
\omega_{*, r}(X)=\bigoplus_{n=0}^{\infty} \omega_{n, r}(X)
$$

is always an $\omega_{*}(k)$-module via product (and pull-back). If $X \in \mathbf{S m}_{k}$, then $\omega_{*, r}(X)$ is also a module over the ring $\omega_{*}(X)$.

\subsection{Basis}

The main result of the paper is the construction of a basis of $\omega_{n, r}(k)$ analogous to the fundamental presentation (0.7). Our basis is indexed by pairs of partitions. A partition pair of size $n$ and type $r$ is a pair $(\lambda, \mu)$ where

(i) $\lambda$ is a partition of $n$,

(ii) $\mu$ is a subpartition of $\lambda$ of length $\ell(\mu) \leq r$.

Being a subpartition means $\mu$ is obtained by deleting parts of $\lambda$. The partition $\mu$ may be empty and may equal $\lambda$ if $\ell(\lambda) \leq r$. Subpartitions $\mu, \mu^{\prime} \subset \lambda$ are equivalent if they differ by permuting equal parts of $\lambda$.

Let $\mathcal{P}_{n, r}$ be the set of all partition pairs of size $n$ and type $r$. For example,

$$
\mathcal{P}_{3,2}=\left\{\begin{array}{c}
(3, \emptyset),(3,3), \\
(21, \emptyset),(21,2),(21,1),(21,21), \\
(111, \emptyset),(111,1),(111,11)
\end{array}\right\} .
$$

To each $(\lambda, \mu) \in \mathcal{P}_{n, r}$, we associate an element $\phi(\lambda, \mu) \in \omega_{n, r}(k)$ by the following construction. Let $\mathbb{P}^{\lambda}=\mathbb{P}^{\lambda_{1}} \times \cdots \times \mathbb{P}^{\lambda_{\ell}(\lambda)}$. To each part $m$ of $\mu$, let $L_{m} \rightarrow \mathbb{P}^{\lambda}$ be the line bundle obtained by pulling back $\mathcal{O}_{\mathbb{P}^{m}}(1)$ via the projection to the factor $\mathbb{P}^{\lambda} \rightarrow \mathbb{P}^{m}$ corresponding to the part $m$. Since $\mu \subset \lambda, m$ is part of $\lambda$. We define

$$
\phi(\lambda, \mu)=\left[\mathbb{P}^{\lambda}, \mathcal{O}^{r-\ell(\mu)} \oplus \bigoplus_{m \in \mu} L_{m}\right] .
$$

The bundle on $\mathbb{P}^{\lambda}$ has a trivial factor of $\operatorname{rank} r-\ell(\mu)$. 
Theorem 1. For $n, r \geq 0$, we have

$$
\omega_{n, r}(k) \otimes_{\mathbb{Z}} \mathbb{Q}=\bigoplus_{(\lambda, \mu) \in \mathcal{P}_{n, r}} \mathbb{Q} \cdot \phi(\lambda, \mu) .
$$

In other words, the elements $\phi(\lambda, \mu)$ determine a basis of $\omega_{n, r}(k) \otimes_{\mathbb{Z}} \mathbb{Q}$. If $r=0$, Theorem 1 specializes to $(0.7)$. In case $(n, r)=(3,2)$, the basis of Theorem 1 is given by

$$
\begin{aligned}
& {\left[\mathbb{P}^{3}, \mathcal{O}^{2}\right], \quad\left[\mathbb{P}^{3}, \mathcal{O} \oplus \mathcal{O}(1)\right],} \\
& {\left[\mathbb{P}^{2} \times \mathbb{P}^{1}, \mathcal{O}^{2}\right], \quad\left[\mathbb{P}^{2} \times \mathbb{P}^{1}, \mathcal{O} \oplus \mathcal{O}(1,0)\right],} \\
& {\left[\mathbb{P}^{2} \times \mathbb{P}^{1}, \mathcal{O} \oplus \mathcal{O}(0,1)\right], \quad\left[\mathbb{P}^{2} \times \mathbb{P}^{1}, \mathcal{O}(1,0) \oplus \mathcal{O}(0,1)\right],} \\
& {\left[\mathbb{P}^{1} \times \mathbb{P}^{1} \times \mathbb{P}^{1}, \mathcal{O}^{2}\right], \quad\left[\mathbb{P}^{1} \times \mathbb{P}^{1} \times \mathbb{P}^{1}, \mathcal{O} \oplus \mathcal{O}(1,0,0)\right],} \\
& {\left[\mathbb{P}^{1} \times \mathbb{P}^{1} \times \mathbb{P}^{1}, \mathcal{O}(1,0,0) \oplus \mathcal{O}(0,1,0)\right] .}
\end{aligned}
$$

Theorem 1 is proven in Section 3. The argument requires studying an algebraic cobordism theory for lists of line bundles on varieties developed in Section 2.

The structure of $\omega_{*, r}(k)$ over $\mathbb{Z}$ is determined by the following result proven in Section 3.3.

Theorem 2. For $r \geq 0, \omega_{*, r}(k)$ is a free $\omega_{*}(k)$-module with basis

$$
\omega_{*, r}(k)=\bigoplus_{\lambda} \omega_{*}(k) \cdot \phi(\lambda, \lambda)
$$

where the sum is over all partitions $\lambda$ of length at most $r$.

\subsection{Over X}

In fact, $\omega_{*, r}(k)$ determines $\omega_{*, r}(X)$ for all $X \in \mathbf{S c h}_{k}$. There is a natural map

$$
\gamma_{X}: \omega_{*}(X) \otimes_{\omega_{*}(k)} \omega_{*, r}(k) \rightarrow \omega_{*, r}(X)
$$

of $\omega_{*}(k)$-modules defined by

$$
\gamma_{X}([Y \stackrel{f}{\rightarrow} X] \otimes \phi(\lambda, \lambda))=\left[Y \times \mathbb{P}^{\lambda} \stackrel{f \circ p_{Y}}{\longrightarrow} X, \mathcal{O}^{r-\ell(\lambda)} \oplus \bigoplus_{m \in \lambda} p_{\mathbb{P}^{\lambda}}^{*}\left(L_{m}\right)\right] .
$$

Here, $\lambda$ has length at most $r$, and $p_{Y}$ and $p_{\mathbb{P}^{\lambda}}$ are the projections of $Y \times \mathbb{P}^{\lambda}$ to $Y$ and $\mathbb{P}^{\lambda}$ respectively.

Theorem 3. For $r \geq 0$ and $X \in \mathbf{S c h}_{k}$, the map $\gamma_{X}$ is an isomorphism of $\omega_{*}(k)$-modules.

By Theorem 3, the algebraic cobordism theory $\omega_{*, r}$ of bundles on varieties is simply an extension of scalars of the original theory $\omega_{*}$. 


\subsection{Chern invariants}

Let $Y$ be a nonsingular projective variety of dimension $n$, and let $E$ be a rank $r$ vector bundle on $Y$. The Chern invariants of the pair $[Y, E]$ are

$$
\int_{Y} \Theta\left(c_{1}\left(T_{Y}\right), \ldots, c_{n}\left(T_{Y}\right), c_{1}(E), \ldots, c_{r}(E)\right)
$$

where $\Theta$ is any graded degree $n$ polynomial (with $\mathbb{Q}$-coefficients) of the Chern classes of the tangent bundle $T_{Y}$ and $E$.

Let $\mathcal{C}_{n, r}$ be the finite-dimensional $\mathbb{Q}$-vector space of graded degree $n$ polynomials in the Chern classes.

Theorem 4. The Chern invariants respect algebraic cobordism. The resulting map

$$
\omega_{n, r}(k) \otimes_{\mathbb{Z}} \mathbb{Q} \rightarrow \mathcal{C}_{n, r}^{*}
$$

is an isomorphism.

A simple counting argument (given in Section 1) shows the dimension of $\mathcal{C}_{n, r}$ equals the cardinality of $\mathcal{P}_{n, r}$. In case $(n, r)=(3,2)$, there are nine basic Chern invariants of $[Y, E]$,

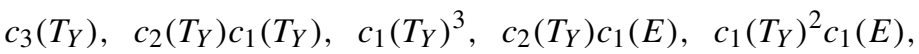

$$
\begin{aligned}
& c_{1}\left(T_{Y}\right) c_{2}(E), \quad c_{1}\left(T_{Y}\right) c_{1}(E)^{2}, \quad c_{2}(E) c_{1}(E), c_{1}(E)^{3} .
\end{aligned}
$$

Theorem 4 is proven jointly with Theorem 1 in Section 3.

\subsection{Applications}

For studying a theory associated to pairs $[Y, E]$ which admits a multiplicative double point degeneration formula, algebraic cobordism $\omega_{n, r}(\mathbb{C})$ is a useful tool. The full theory can be calculated from the toric basis elements specified by Theorem 1 .

The determinations of $\omega_{3}(\mathbb{C})$ and $\omega_{2,1}(\mathbb{C})$ have been used in [7] to prove the conjectures of $[1,9,10]$ governing the degrees of virtual classes on the Hilbert schemes of points of 3-folds. Recently, Y. Tzeng [13] has used the 4-dimensional basis of $\omega_{2,1}(\mathbb{C})$ in a beautiful proof of Göttsche's conjecture [3] governing nodal curve counting (interpreted as degrees of cycles in the Hilbert schemes of points of surfaces). The basis of $\omega_{n, r}(\mathbb{C})$ will be used in [5] for the study of flop invariance of quantum cohomology.

\subsection{Speculations}

Consider the algebraic group $\mathbf{G L}_{r}$ over $\mathbb{C}$. We view $\omega_{*, r}(\mathbb{C})$ as an algebraic model for $M U_{*}\left(B \mathbf{G L}_{r}\right)$. Theorem 4 may be interpreted as saying $\omega_{*, r}(\mathbb{C})$ is dual to

$$
M U^{*}\left(B \mathbf{G L}_{r}\right)=M U^{*}(\mathrm{pt})\left[\left[c_{1}, \ldots, c_{r}\right]\right] .
$$

D. Maulik suggests defining an algebraic cobordism theory $\omega_{*, \mathbf{G}}$ for principal G-bundles on algebraic varieties by the double point relation of Definition 3. Perhaps the resulting theory over a point for classical groups $\mathbf{G}$ is dual to $M U^{*}(B \mathbf{G})$ ? 
An algebraic approach to $M U^{*}(B \mathbf{G})$ for linear algebraic groups has been proposed in [2] by limits of $\Omega^{*}$ over algebraic approximations to $B \mathbf{G}$. The construction is similar to Totaro's definition [12] of the Chow ring of $B \mathbf{G}$, but requires also the coniveau filtration (see [8] for an alternative limit definition). For many examples, including $B \mathbf{G L}_{r}$, the isomorphism

$$
\Omega^{*}(B \mathbf{G}) \cong M U^{*}(B \mathbf{G})
$$

is obtained [2]. Such isomorphisms were predicted in [14].

Another approach to our paper is perhaps possible via a limit definition of $\Omega_{*}\left(B \mathbf{G} \mathbf{L}_{r}\right)$. There should be a map

$$
\Omega_{*}\left(B \mathbf{G L}_{r}\right) \rightarrow \omega_{*, r}(\mathbb{C})
$$

which is injective by Chern invariants and surjective by Proposition 11.

\section{Chern classes}

\subsection{Cobordism invariance}

Let $n, r \geq 0$. There is a canonical bilinear map

$$
\rho: \mathcal{M}_{n, r}(k)^{+} \otimes_{\mathbb{Z}} \mathbb{Q} \times \mathcal{C}_{n, r} \rightarrow \mathbb{Q}
$$

defined by integration,

$$
\rho([Y, E], \Theta)=\int_{Y} \Theta\left(c_{1}\left(T_{Y}\right), \ldots, c_{n}\left(T_{Y}\right), c_{1}(E), \ldots, c_{r}(E)\right) .
$$

Proposition 5. The pairing $\rho$ annihilates $\mathcal{R}_{n, r}(k)$.

Proof. In case $r=0$, the invariance of the Chern numbers of the tangent bundle is a well-known property of algebraic cobordism over a $\operatorname{Spec}(\mathbb{C})($ see $[6,11])$.

Let $Y \in \mathbf{S m}_{k}$ be of pure dimension $n+1$. Let

$$
\pi: Y \rightarrow \mathbb{P}^{1}
$$

be a projective morphism which is a double point degeneration over $0 \in \mathbb{P}^{1}$. Let $L$ be a line bundle on $Y$. Suppose $L$ is very ample on $Y$. Cutting $Y$ with $s$ generic sections of $L$ yields a nonsingular subvariety of codimension $s$,

$$
D^{s} \subset Y \stackrel{\pi}{\rightarrow} \mathbb{P}^{1}
$$

The composition $D^{s} \rightarrow \mathbb{P}^{1}$ is a double point degeneration over $0 \in \mathbb{P}^{1}$.

Let $Y_{\zeta}, A, B$, and $\mathbb{P}(\pi)$ be the four spaces which occur in the double point relation for (1.1) in Definition 3. Let

$$
Y_{\zeta} \cap D^{s}, \quad A \cap D^{s}, \quad B \cap D^{s}, \mathbb{P}(\pi) \cap D^{s}
$$


be the four spaces which occur in the relation for $D^{s} \rightarrow \mathbb{P}^{1}$. Since the tangent bundle of $Z \cap D^{s}$ satisfies

$$
\left.0 \rightarrow T_{Z \cap D^{s}} \rightarrow T_{Z}\right|_{Z \cap D^{s}} \rightarrow \bigoplus_{i=1}^{s} L \rightarrow 0
$$

in each of the four cases, we have

$$
c\left(T_{Z \cap D^{s}}\right)=\frac{c\left(T_{Z}\right)}{\left(1+c_{1}(L)\right)^{s}}, \quad c_{i}\left(T_{Z \cap D^{s}}\right)=c_{i}\left(T_{Z}\right)-s \cdot c_{i-1}\left(T_{Z}\right) c_{1}(L)+\cdots,
$$

where we have suppressed the restrictions. The application of the $r=0$ case of the proposition to the degenerations $D^{s} \rightarrow \mathbb{P}^{1}$ for all $s$ implies (by descending induction) the $r=1$ case for double point relations where $L$ is ample.

Similarly if $L_{1}, \ldots, L_{m}$ are very ample line bundles on $Y$, we can consider

$$
D^{s_{1}, \ldots, s_{m}} \subset Y \stackrel{\pi}{\rightarrow} \mathbb{P}^{1}
$$

obtained by cutting with $s_{1}$ sections of $L_{1}, s_{2}$ sections of $L_{2}, \ldots$, and $s_{m}$ sections of $L_{m}$. The application of the $r=0$ case of the proposition to the degeneration $D^{s_{1}, \ldots, s_{m}} \rightarrow \mathbb{P}^{1}$ for all $s_{1}, \ldots, s_{m}$ implies invariance under the double point relation of graded degree $n$ polynomials in the Chern classes of the tangent bundle and the Chern classes of $L_{1}, \ldots, L_{m}$.

The $r=1$ case of the proposition follows since every line bundle $L$ may be written as the difference of two very ample line bundles.

To prove the $r>1$ case, we use a splitting argument. Let $\pi$ be a double point degeneration (1.1). Let $E$ be a rank $r$ bundle on $Y$. Let

$$
\mathbb{F}(E) \rightarrow Y \stackrel{\pi}{\rightarrow} \mathbb{P}^{1}
$$

by the complete flag variety over $Y$ obtained from $E$. The composition $\mathbb{F}(E) \rightarrow \mathbb{P}^{1}$ is a double point degeneration with tautological line bundles $L_{1}, \ldots, L_{r}$ which sum in $K$ theory to the pull-back of $E$. The established line bundle results then yield the $r>1$ case.

As a consequence of Proposition 5, the pairing $\rho$ descends,

$$
\rho: \omega_{n, r}(k) \otimes_{\mathbb{Z}} \mathbb{Q} \times \mathcal{C}_{n, r} \rightarrow \mathbb{Q} .
$$

Our first goal is to bound the rank of the pairing from below.

\subsection{Independence}

1.2.1. Monomials of $\mathcal{C}_{n, r}$. For notational convenience, we write elements $\Theta \in \mathcal{C}_{n, r}$ as polynomials

$$
\Theta\left(u_{1}, \ldots, u_{n}, v_{1}, \ldots, v_{r}\right)
$$

where $u_{i}=c_{i}\left(T_{Y}\right)$ and $v_{i}=c_{i}(E)$. Both $u_{i}$ and $v_{i}$ have degree $i$. A canonical basis of $\mathcal{C}_{n, r}$ is obtained from monomials of graded degree $n$.

Let $\mathcal{Q}_{n, r}$ be the set of partition pairs $(\nu, \mu)$ where

(i) $\mu$ is a partition of size $|\mu| \leq n$ with largest part at most $r$,

(ii) $\nu$ is a partition of $n-|\mu|$. 
The correspondence

$$
\prod_{i=1}^{n} u_{i}^{l_{i}} \prod_{j=1}^{r} v_{j}^{m_{j}} \leftrightarrow\left(1^{l_{1}} \cdots n^{l_{n}}, 1^{m_{1}} \cdots r^{m_{r}}\right)
$$

yields a bijection between the monomial basis of $\mathcal{C}_{n, r}$ and the set $\mathcal{Q}_{n, r}$. Let $\mathrm{C}(\nu, \mu)$ denote the monomial associated to $(\nu, \mu) \in \mathcal{Q}_{n, r}$.

Lemma 6. There is a natural bijection $\epsilon: \mathcal{Q}_{n, r} \rightarrow \mathcal{P}_{n, r}$.

Proof. Given $(\nu, \mu) \in \mathcal{Q}_{n, r}$, define $\epsilon(\nu, \mu)=\left(v \cup \mu^{t}, \mu^{t}\right) \in \mathcal{P}_{n, r}$. Here, $\mu^{t}$ is the partition obtained by transposing the Young diagram associated to $\mu$. Hence, $\mu^{t}$ has length at most $r$.

1.2.2. Ordering. The $v$-degree of a monomial in $\mathcal{C}_{n, r}$ is the vector

$$
\operatorname{deg}_{v}\left(\prod_{i=1}^{n} u_{i}^{l_{i}} \prod_{j=1}^{r} v_{j}^{m_{j}}\right)=\left(m_{1}, \ldots, m_{r}\right) \in \mathbb{Z}_{\geq 0}^{r}
$$

We define a total ordering on $\mathbb{Z}_{\geq 0}^{r}$ by the following rule: $\left(m_{1}, \ldots, m_{r}\right)>\left(m_{1}^{\prime}, \ldots, m_{r}^{\prime}\right)$ if either $m_{r}>m_{r}^{\prime}$, or $m_{j}=m_{j}^{\prime}$ for all $j>i$ and $m_{i}>m_{i}^{\prime}$. The resulting partial order on the monomials on $\mathcal{C}_{n, r}$ (indexed by $\mathcal{Q}_{n, r}$ ) is sensitive only to the variables $v_{i}$.

1.2.3. Bilinear pairing. Let $\mathbf{M}$ be the matrix with rows and columns indexed by $\mathcal{Q}_{n, r}$ and elements

$$
\mathbf{M}_{n, r}\left[(v, \mu),\left(v^{\prime}, \mu^{\prime}\right)\right]=\rho\left(\phi(\epsilon(v, \mu)), \mathrm{C}\left(v^{\prime}, \mu^{\prime}\right)\right)
$$

for $(\nu, \mu),\left(\nu^{\prime}, \mu^{\prime}\right) \in \mathcal{Q}_{n, r}$. Recall that the map $\phi: \mathcal{P}_{n, r} \rightarrow \omega_{n, r}(k)$ was defined in Section 0.7. The rows and columns of $\mathbf{M}_{n, r}$ are ordered by the partial ordering on $\mathcal{Q}_{n, r}$ defined in Section 1.2.2.

Lemma 7. If $(v, \mu)<\left(v^{\prime}, \mu^{\prime}\right)$ in the partial order of $\mathcal{Q}_{n, r}$, then

$$
\mathbf{M}_{n, r}\left[(\nu, \mu),\left(v^{\prime}, \mu^{\prime}\right)\right]=0 .
$$

Proof. Let $\mu=1^{m_{1}} \cdots r^{m_{r}}$ and $\mu^{\prime}=1^{m_{1}^{\prime}} \ldots r^{m_{r}^{\prime}}$. If $(\nu, \mu)<\left(v^{\prime}, \mu^{\prime}\right)$, then, at the highest index $i$ where a difference occurs, $m_{i}<m_{i}^{\prime}$.

Suppose the difference occurs at $i=r$. Then $m_{r}$ is the minimal part of $\mu^{t}$. For the pair $[Y, E]=\phi(v, \mu)$, the bundle $E$ is a direct sum of $r$ line bundles pulled back from the $\mathcal{O}(1)$ factors of a product of $r$ projective spaces (with minimal dimension $m_{r}$ ). Since $m_{r}<m_{r}^{\prime}$, the class $c_{r}^{m_{r}^{\prime}}(E)$ vanishes on $Y$ by dimensional considerations.

If the highest difference occurs at $i<r$, the argument is the same (following again from elementary dimensional considerations).

Proposition 8. $\mathbf{M}_{n, r}$ is a nonsingular matrix. 
Proof. By Lemma 7, the matrix $\mathbf{M}_{n, r}$ is block lower triangular with respect to the partial ordering on $\mathcal{Q}_{n, r}$. The blocks are determined by all $(\nu, \mu) \in \mathcal{Q}_{n, r}$ with the same $\mu$.

Let $\mu=1^{m_{1}} \ldots r^{m_{r}}$. Consider the bundle

$$
E=\bigoplus_{m \in \mu^{t}} L_{m} \rightarrow \mathbb{P}^{\mu^{t}}
$$

following the notation of Section 0.7. Since

$$
\int_{\mathbb{P}^{\mu^{t}}} c_{1}(E)^{m_{1}} \ldots c_{r}(E)^{m_{r}}=1
$$

the block in $\mathbf{M}_{n, r}$ corresponding to $\mu$ is the matrix $\mathbf{M}_{n-|\mu|, 0}$. The latter is nonsingular by well-known results about the usual $r=0$ theory of algebraic cobordism $[6,11]$.

As a consequence of Proposition 8, the generators proposed in Theorem 1 span a subspace of $\omega_{n, r}(k) \otimes_{\mathbb{Z}} \mathbb{Q}$ of rank at least $\left|\mathcal{P}_{n, r}\right|$. In particular,

$$
\operatorname{dim}\left(\omega_{n, r}(k) \otimes_{\mathbb{Z}} \mathbb{Q}\right) \geq\left|\mathcal{P}_{n, r}\right| .
$$

Moreover, the pairing (1.2) has rank at least $\left|\mathcal{P}_{n, r}\right|$. To complete the proofs of Theorem 1 and 4 , we will prove the reverse inequality $\operatorname{dim}\left(\omega_{n, r}(k) \otimes_{\mathbb{Z}} \mathbb{Q}\right) \leq\left|\mathcal{P}_{n, r}\right|$ in Section 3 .

\section{Lists of line bundles}

\subsection{Lists}

For $X \in \mathbf{S c h}_{k}$, let $\mathcal{M}_{n, 1^{r}}(X)$ denote the set of isomorphism classes over $X$ of tuples [ $f: Y \rightarrow X, L_{1}, \ldots, L_{r}$ ] with $Y \in \mathbf{S m}_{k}$ of dimension $n, f$ projective, and $L_{1}, \ldots, L_{r}$ an ordered list of line bundles on $Y$. The set $\mathcal{M}_{n, 1^{r}}(X)$ is a monoid under disjoint union of domains. Let $\mathcal{M}_{n, 1^{r}}(X)^{+}$denote the group completion of $\mathcal{M}_{n, 1^{r}}(X)$.

Let $Y \in \mathbf{S m}_{k}$ be of pure dimension $n+1$, and let $g: Y \rightarrow X \times \mathbb{P}^{1}$ be a projective morphism for which the composition $\pi=p_{2} \circ g: Y \rightarrow \mathbb{P}^{1}$ is a double point degeneration over $0 \in \mathbb{P}^{1}$. Let $L_{1}, \ldots, L_{r}$ be a list of line bundles on $Y$. Let

$$
\begin{aligned}
{\left[A \rightarrow X, L_{1, A}, \ldots L_{r, A}\right],[B \rightarrow} & \left.X, L_{1, B} \ldots L_{r, B}\right], \\
& {\left[\mathbb{P}(\pi) \rightarrow X, L_{1, \mathbb{P}(\pi)}, \ldots, L_{1, \mathbb{P}(\pi)}\right] \in \mathcal{M}_{n, 1^{r}}(X)^{+} }
\end{aligned}
$$

be obtained from the fiber $\pi^{-1}(0)$ and the morphism $p_{1} \circ g$.

Definition 4. Let $\zeta \in \mathbb{P}^{1}(k)$ be a regular value of $\pi$. The associated double point relation over $X$ is

$$
\left[Y_{\zeta} \rightarrow X,\left\{L_{i, Y_{\zeta}}\right\}\right]-\left[A \rightarrow X,\left\{L_{i, A}\right\}\right]-\left[B \rightarrow X,\left\{L_{i, B}\right\}\right]+\left[\mathbb{P}(\pi) \rightarrow X,\left\{L_{i, \mathbb{P}(\pi)}\right\}\right]
$$

where $Y_{\zeta}=\pi^{-1}(\zeta)$ 
For $X \in \mathbf{S c h}_{k}$, let $\mathcal{R}_{n, 1^{r}}(X) \subset M_{n, 1^{r}}(X)^{+}$be the subgroup generated by all double point relations. Double point cobordism theory for lists of line bundles on varieties is defined by

$$
\omega_{n, 1^{r}}(X)=\mathcal{M}_{n, 1^{r}}(X)^{+} / \mathcal{R}_{n, 1^{r}}(X)
$$

The sum

$$
\omega_{*, 1^{r}}(X)=\bigoplus_{n=0}^{\infty} \omega_{n, 1^{r}}(X)
$$

is always an $\omega_{*}(k)$-module via product. If $X \in \mathbf{S m}_{k}$, then $\omega_{*, 1^{r}}(X)$ is also a module over the $\operatorname{ring} \omega_{*}(X)$.

\subsection{Basis}

A partition list of size $n$ and type $r$ is a tuple $\left(\lambda,\left(m_{1}, \ldots, m_{r}\right)\right)$ where

(i) $\lambda$ is a partition of $n$,

(ii) $\left(m_{1}, \ldots, m_{r}\right)$ is a list with $m_{i} \geq 0$ whose union of non-zero parts is a subpartition $\mu \subset \lambda$.

Let $\mathcal{P}_{n, 1^{r}}$ be the set of all partition lists of size $n$ and type $r$. For example,

$$
\mathcal{P}_{3,1^{2}}=\left\{\begin{array}{c}
(3,(0,0)),(3,(3,0)),(3,(0,3)) \\
(21,(0,0)),(21,(2,0)),(21,(1,0)) \\
(21,(0,1)),(21,(0,2)),(21,(2,1)),(21,(1,2)) \\
(111,(0,0)),(111,(1,0)),(111,(0,1)),(111,(1,1))
\end{array}\right\} .
$$

To each $\left(\lambda,\left(m_{1}, \ldots, m_{r}\right)\right) \in \mathcal{P}_{n, 1^{r}}$, we associate an element

$$
\phi\left(\lambda,\left(m_{1}, \ldots, m_{r}\right)\right) \in \omega_{n, r}(k)
$$

by the following construction. Let $\mathbb{P}^{\lambda}=\mathbb{P}^{\lambda_{1}} \times \ldots \times \mathbb{P}^{\lambda_{\ell(\lambda)}}$. To each non-zero part $m_{i}$, let $L_{m_{i}} \rightarrow \mathbb{P}^{\lambda}$ be the line bundle obtained by pulling back $\mathcal{O}_{\mathbb{P}^{m_{i}}}(1)$ via the projection to the factor $\mathbb{P}^{\lambda} \rightarrow \mathbb{P}^{m_{i}}$ corresponding to the part $m_{i}$. If $m_{i}=0$, let $L_{m_{i}}$ be the trivial line bundle on $\mathbb{P}^{\lambda}$. We define

$$
\phi\left(\lambda,\left(m_{1}, \ldots, m_{r}\right)\right)=\left[\mathbb{P}^{\lambda},\left(L_{m_{1}}, \ldots, L_{m_{r}}\right)\right] .
$$

Theorem 9. For $n, r \geq 0$, we have

$$
\omega_{n, 1^{r}} \otimes_{\mathbb{Z}} \mathbb{Q}=\bigoplus_{\left(\lambda,\left(m_{1}, \ldots, m_{r}\right)\right) \in \mathcal{P}_{n, 1^{r}}} \mathbb{Q} \cdot \phi\left(\lambda,\left(m_{1}, \ldots, m_{r}\right)\right) .
$$

Theorem 9 will be proven in Section 2.6 with a mix of techniques from $[6,7]$ and new methods for studying algebraic cobordism relations for line bundles on varieties. 


\subsection{Chern invariants}

Let $\mathcal{C}_{n, 1^{r}}$ be the $\mathbb{Q}$-vector space of graded degree $n$ polynomials in the Chern classes $c_{1}\left(T_{Y}\right), \ldots, c_{n}\left(T_{Y}\right), c_{1}\left(L_{1}\right), \ldots, c_{1}\left(L_{r}\right)$. There is a canonical bilinear map

$$
\rho: \mathcal{M}_{n, 1^{r}}(k)^{+} \otimes_{\mathbb{Z}} \mathbb{Q} \times \mathcal{C}_{n, 1^{r}} \rightarrow \mathbb{Q}
$$

defined by integration,

$$
\rho([Y, E], \Theta)=\int_{Y} \Theta\left(c_{1}\left(T_{Y}\right), \ldots, c_{n}\left(T_{Y}\right), c_{1}\left(L_{1}\right), \ldots, c_{1}\left(L_{r}\right)\right) .
$$

The proof of Proposition 5 implies the pairing $\rho$ annihilates $\mathcal{R}_{n, 1^{r}}(k)$. Hence, $\rho$ descends,

$$
\rho: \omega_{n, 1^{r}}(k) \otimes_{\mathbb{Z}} \mathbb{Q} \times \mathcal{C}_{n, 1^{r}} \rightarrow \mathbb{Q} .
$$

The monomial basis of $\mathcal{C}_{n, 1^{r}}$ is easily seen to have the same cardinality as the set $\mathcal{P}_{n, 1^{r}}$. A straightforward extension of the methods of Section 1.2.3 implies that the elements of

$$
\left\{\phi\left(\lambda,\left(m_{1}, \ldots, m_{r}\right)\right) \mid\left(\lambda,\left(m_{1}, \ldots, m_{r}\right)\right) \in \mathcal{P}_{n, 1^{r}}\right\} \subset \omega_{n, 1^{r}} \otimes_{\mathbb{Z}} \mathbb{Q}
$$

span a subspace of dimension $\left|\mathcal{P}_{n, 1^{r}}\right|$. In particular,

$$
\operatorname{dim}\left(\omega_{n, 1^{r}} \otimes_{\mathbb{Z}} \mathbb{Q}\right) \geq\left|\mathcal{P}_{n, 1^{r}}\right| .
$$

\subsection{Globally generated line bundles}

Let $\mathfrak{m} \subset \omega_{*}(k)$ be the ideal generated by all elements of positive dimension,

$$
0 \rightarrow \mathfrak{m} \rightarrow \omega_{*}(k) \rightarrow \mathbb{Z} \rightarrow 0 .
$$

Since $\omega_{*, 1^{r}}(k)$ is an $\omega_{*}(k)$-module, we can define the graded quotient

$$
\widetilde{\omega}_{*, 1^{r}}(k)=\frac{\omega_{*, 1^{r}}(k)}{\mathfrak{m} \cdot \omega_{*, 1^{r}}(k)}, \quad \widetilde{\omega}_{*, 1^{r}}(k)=\bigoplus_{n=0}^{\infty} \widetilde{\omega}_{n, 1^{r}}(k) .
$$

For $\left(\lambda,\left(m_{1}, \ldots, m_{r}\right)\right) \in \mathcal{P}_{n, 1^{r}}$, let

$$
\widetilde{\phi}\left(\lambda,\left(m_{1}, \ldots, m_{r}\right)\right) \in \widetilde{\omega}_{n, 1^{r}}(k)
$$

denote the class of $\phi\left(\lambda,\left(m_{1}, \ldots, m_{r}\right)\right)$ in the quotient.

Proposition 10. Let $Y \in \mathbf{S m}_{k}$ be a projective variety of dimension $n$ with line bundles $L_{1}, \ldots, L_{r}$ all generated by global sections. Then $\left[Y, L_{1}, \ldots, L_{r}\right] \in \widetilde{\omega}_{n, 1^{r}}(k)$ lies in the $\mathbb{Z}$-linear span of

$$
\left\{\widetilde{\phi}\left(\lambda,\left(m_{1}, \ldots, m_{r}\right)\right) \mid\left(\lambda,\left(m_{1}, \ldots, m_{r}\right)\right) \in \mathcal{P}_{n, 1^{r}}, \sum_{i=1}^{r} m_{i}=n\right\}
$$

in $\widetilde{\omega}_{n, 1^{r}}(k)$. 
Proof. Since $L_{1}, \ldots, L_{r}$ are all generated by global sections on $Y$, there exists a projective morphism

$$
f: Y \rightarrow \mathbb{P}^{d_{1}} \times \cdots \times \mathbb{P}^{d_{r}}, \quad L_{i}=f^{*}\left(\mathcal{O}_{\mathbb{P}^{d_{i}}}(1)\right) .
$$

We view $f$ as determining an element of algebraic cobordism,

$$
\left[f: Y \rightarrow \mathbb{P}^{d_{1}} \times \cdots \times \mathbb{P}^{d_{r}}\right] \in \omega_{n}\left(\mathbb{P}^{d_{1}} \times \cdots \times \mathbb{P}^{d_{r}}\right) .
$$

A fundamental result of [6, Theorem 1.2.19] is the isomorphism

$$
A_{*}(X) \cong \widetilde{\omega}_{*}(X)=\omega_{*}(X) / \mathfrak{m} \cdot \omega_{*}(X),
$$

where $A_{*}(X)$ is the Chow theory of $X$ (with $\mathbb{Z}$ coefficients). The Chow group

$$
A_{n}\left(\mathbb{P}^{d_{1}} \times \cdots \times \mathbb{P}^{d_{r}}\right)
$$

is generated by linear subvarieties

$$
\iota_{m_{1}, \ldots, m_{r}}: \mathbb{P}^{m_{1}} \times \cdots \times \mathbb{P}^{m_{r}} \hookrightarrow \mathbb{P}^{d_{1}} \times \cdots \times \mathbb{P}^{d_{r}}
$$

where $\sum_{i=1}^{r} m_{i}=n$. We conclude $[f]$ is a $\mathbb{Z}$-linear combination of the elements

$$
\left[\iota_{m_{1}, \ldots, m_{r}}\right] \in \widetilde{\omega}_{n}\left(\mathbb{P}^{d_{1}} \times \cdots \times \mathbb{P}^{d_{r}}\right) .
$$

Relations in $\omega_{n}\left(\mathbb{P}^{d_{1}} \times \cdots \times \mathbb{P}^{d_{r}}\right)$ lift canonically to $\omega_{n, 1^{r}}\left(\mathbb{P}^{d_{1}} \times \cdots \times \mathbb{P}^{d_{r}}\right)$ by pulling back the list

$$
\mathcal{O}_{\mathbb{P}^{d_{1}}}(1), \ldots, \mathcal{O}_{\mathbb{P} d_{r}}(1)
$$

everywhere. Since all double point relations in $\omega_{n}\left(\mathbb{P}^{d_{1}} \times \cdots \times \mathbb{P}^{d_{r}}\right)$ occur over $\mathbb{P}^{d_{1}} \times \cdots$ $\times \mathbb{P}^{d_{r}}$, the pull-back of the list (2.2) is well-defined and canonical. The pull-back of the list (2.2) via $\iota_{m_{1}, \ldots, m_{r}}$ yields the element

$$
\phi\left(\lambda,\left(m_{1}, \ldots, m_{r}\right)\right) \in \omega_{n, 1^{r}}(k),
$$

where $\sum_{i=1}^{r} m_{i}=n$. Here, $\lambda$ is obtained simply by removing the 0 parts $m_{i}$. Hence, after pushing forward from $\mathbb{P}^{d_{1}} \times \cdots \times \mathbb{P}^{d_{r}}$ to $\operatorname{Spec}(k)$, the argument is complete.

\subsection{Projective bundles}

We will need auxiliary results on projective bundles to remove the global generation hypothesis of Proposition 10.

Let $Z \in \mathbf{S m}_{k}$ be a projective variety equipped with a list of line bundles $L_{1}, \ldots, L_{r}$ and a split rank 2 vector bundle

$$
B=\mathcal{O}_{Z} \oplus N .
$$

We are interested in the classes

$$
\left[\mathbb{P}(B), L_{1}, \ldots, L_{r}\right],\left[\mathbb{P}(B), L_{1}( \pm 1), \ldots, L_{r}( \pm 1)\right] \in \omega_{*, 1^{r}}(k) .
$$

Here, $\mathbb{P}(B)$ denotes the projectivization by sublines, and $L_{i}( \pm 1)$ stands for $L_{i} \otimes$ $\mathcal{O}_{\mathbb{P}(B)}( \pm 1)$. 
Let $s$ be the section $Z \rightarrow \mathbb{P}(B)$ determined by the factor $N \subset B$. The divisor $s$ is an element of the linear series associated to $\mathcal{O}_{\mathbb{P}(B)}(1)$. The degeneration to the normal cone of $s$ yields a double point relation in $\omega_{*}(Z)$. After pulling back the list $L_{1}, \ldots, L_{r}$, we obtain a double point relation in $\omega_{*, 1^{r}}(Z)$. Twisting the list by the exceptional divisor of the degeneration yields the relation

$$
\begin{array}{r}
{\left[\mathbb{P}(B), L_{1}, \ldots, L_{r}\right]-\left[\mathbb{P}(B), L_{1}(1), \ldots, L_{r}(1)\right]-\left[\mathbb{P}\left(\mathcal{O}_{Z} \oplus N^{*}\right), L_{1}(-1), \ldots L_{r}(-1)\right]} \\
+\left[\mathbb{P}(B), L_{1} \otimes N^{*}, \ldots, L_{r} \otimes N^{*}\right]=0 \text { in } \omega_{*, 1^{r}}(Z) .
\end{array}
$$

Since $\left(\mathcal{O}_{Z} \oplus N^{*}\right) \otimes N \cong B$, we may rewrite the above relation in the following form:

$$
\begin{array}{r}
{\left[\mathbb{P}(B), L_{1}, \ldots, L_{r}\right]-\left[\mathbb{P}(B), L_{1}(1), \ldots, L_{r}(1)\right]-\left[\mathbb{P}(B), L_{1} \otimes N^{*}(-1), \ldots L_{r} \otimes N^{*}(-1)\right]} \\
+\left[\mathbb{P}(B), L_{1} \otimes N^{*}, \ldots, L_{r} \otimes N^{*}\right]=0 \quad \text { in } \omega_{*, 1^{r}}(Z) .
\end{array}
$$

After replacing $L_{i}$ with $L_{i} \otimes N$ everywhere, we obtain our main projective bundle relation in $\omega_{*, 1^{r}}(Z)$ :

$$
\begin{aligned}
{\left[\mathbb{P}(B), L_{1}(-1), \ldots L_{r}(-1)\right]=} & {\left[\mathbb{P}(B), L_{1} \otimes N, \ldots, L_{r} \otimes N\right] } \\
& -\left[\mathbb{P}(B), L_{1} \otimes N(1), \ldots, L_{r} \otimes N(1)\right] \\
& +\left[\mathbb{P}(B), L_{1}, \ldots, L_{r}\right] .
\end{aligned}
$$

Proposition 11. Let $Y \in \mathbf{S m}_{k}$ be a projective variety of dimension $n$ with arbitrary line bundles $L_{1}, \ldots, L_{r}$. Then $\left[Y, L_{1}, \ldots, L_{r}\right] \in \widetilde{\omega}_{n, 1^{r}}(k)$ lies in the $\mathbb{Z}$-linear span of

$$
\left\{\widetilde{\phi}\left(\lambda,\left(m_{1}, \ldots, m_{r}\right)\right) \mid\left(\lambda,\left(m_{1}, \ldots, m_{r}\right)\right) \in \mathcal{P}_{n, 1^{r}}, \sum_{i=1}^{r} m_{i}=n\right\}
$$

in $\widetilde{\omega}_{n, 1^{r}}(k)$.

Proof. Let $Z \subset Y$ be a nonsingular divisor such that $L_{1}(Z), \ldots, L_{r}(Z)$ are all globally generated. Consider the double point relation in $\omega_{n, 1^{r}}(Y)$ obtained from degenerating to the normal cone of $Z$, pulling back the list $L_{1}, \ldots, L_{r}$, and twisting by the exceptional divisor of the degeneration:

$$
\begin{aligned}
{\left[Y, L_{1}, \ldots, L_{r}\right]-} & {\left[Y, L_{1}(Z), \ldots, L_{r}(Z)\right]-\left[\mathbb{P}\left(\mathcal{O}_{Z} \oplus \mathcal{O}_{Z}(Z)\right), L_{1}(-1), \ldots L_{r}(-1)\right] } \\
+ & {\left[\mathbb{P}\left(\mathcal{O}_{Z} \oplus \mathcal{O}_{Z}(Z)\right), L_{1}(Z), \ldots, L_{r}(Z)\right]=0 \quad \text { in } \omega_{n, 1^{r}}(Y) . }
\end{aligned}
$$

Proposition 10 applies to the second and fourth terms of relation (2.3). The third term, however, requires further analysis. Using our main projective bundle relation in $\omega_{n, 1^{r}}(Z)$, we can trade the third term for

$$
\begin{array}{r}
-\left[\mathbb{P}\left(\mathcal{O}_{Z} \oplus \mathcal{O}_{Z}(Z)\right), L_{1}(Z), \ldots, L_{r}(Z)\right]+\left[\mathbb{P}\left(\mathcal{O}_{Z} \oplus \mathcal{O}_{Z}(Z)\right), L_{1}(Z)(1), \ldots, L_{r}(Z)(1)\right] \\
-\left[\mathbb{P}\left(\mathcal{O}_{Z} \oplus \mathcal{O}_{Z}(Z)\right), L_{1}, \ldots, L_{r}\right]
\end{array}
$$

The last two terms are not covered by Proposition 10. 
We have proven the proposition modulo elements of the form

$$
\left[\mathbb{P}(B), L_{1}^{\prime}, \ldots, L_{r}^{\prime}\right],\left[\mathbb{P}(B), L_{1}^{\prime}(1), \ldots, L_{r}^{\prime}(1)\right] \in \omega_{n, 1^{r}}(Z)
$$

where $B=\mathcal{O}_{Z} \oplus N$ is a split rank 2 bundle and $L_{i}^{\prime}$ are arbitrary line bundles on $Z$. Let $\pi: \mathbb{P}(B) \rightarrow Z$ be the projection. Let $Z^{\prime} \subset Z$ be a nonsingular divisor such that

$$
L_{1}^{\prime}\left(Z^{\prime}\right), \ldots, L_{r}^{\prime}\left(Z^{\prime}\right), L_{1}^{\prime}\left(Z^{\prime}\right)(1), \ldots, L_{r}^{\prime}\left(Z^{\prime}\right)(1)
$$

are all globally generated on $\mathbb{P}(B)$.

Consider the double point relation in $\omega_{n, 1^{r}}(Z)$ obtained from degenerating to the normal cone of $\pi^{-1}\left(Z^{\prime}\right) \subset \mathbb{P}(B)$, pulling back the list $L_{1}^{\prime}, \ldots, L_{r}^{\prime}$, and twisting by the exceptional divisor of the degeneration:

$$
\begin{aligned}
& {\left[\mathbb{P}(B), L_{1}^{\prime}, \ldots, L_{r}^{\prime}\right]-\left[\mathbb{P}(B), L_{1}^{\prime}\left(Z^{\prime}\right), \ldots, L_{r}^{\prime}\left(Z^{\prime}\right)\right]} \\
& \quad-\left[\mathbb{P}\left(B_{Z^{\prime}}\right) \times_{Z^{\prime}} \mathbb{P}\left(\mathcal{O}_{Z^{\prime}} \oplus \mathcal{O}_{Z^{\prime}}\left(Z^{\prime}\right)\right), L_{1}^{\prime}(0,-1), \ldots L_{r}^{\prime}(0,-1)\right] \\
& \quad+\left[\mathbb{P}\left(B_{Z^{\prime}}\right) \times_{Z^{\prime}} \mathbb{P}\left(\mathcal{O}_{Z^{\prime}} \oplus \mathcal{O}_{Z^{\prime}}\left(Z^{\prime}\right)\right), L_{1}^{\prime}\left(Z^{\prime}\right), \ldots, L_{r}^{\prime}\left(Z^{\prime}\right)\right]=0
\end{aligned}
$$

in $\omega_{n, 1^{r}}(Z)$. A similar relation holds for $\left[\mathbb{P}(B), L_{1}^{\prime}(1), \ldots, L_{r}^{\prime}(1)\right]$. We treat the third term of (2.4) in both cases with our main projective bundle relation for the $\mathbb{P}\left(\mathcal{O}_{Z^{\prime}} \oplus \mathcal{O}_{Z^{\prime}}\left(Z^{\prime}\right)\right)$ projectivization.

We have now proven the proposition modulo elements of the form

$$
\begin{gathered}
{\left[\mathbb{P}\left(B_{1}\right) \times{ }_{Z^{\prime}} \mathbb{P}\left(B_{2}\right), L_{1}^{\prime \prime}, \ldots, L_{r}^{\prime \prime}\right],} \\
{\left[\mathbb{P}\left(B_{1}\right) \times_{Z^{\prime}} \mathbb{P}\left(B_{2}\right), L_{1}^{\prime \prime}(1,0), \ldots, L_{r}^{\prime \prime}(1,0)\right],} \\
{\left[\mathbb{P}\left(B_{1}\right) \times{ }_{Z^{\prime}} \mathbb{P}\left(B_{2}\right), L_{1}^{\prime \prime}(0,1), \ldots, L_{r}^{\prime \prime}(0,1)\right],} \\
{\left[\mathbb{P}\left(B_{1}\right) \times_{Z^{\prime}} \mathbb{P}\left(B_{2}\right), L_{1}^{\prime \prime}(1,1), \ldots, L_{r}^{\prime \prime}(1,1)\right] \in \omega_{n, 1^{r}}\left(Z^{\prime}\right)}
\end{gathered}
$$

where $B_{i}=\mathcal{O}_{Z^{\prime}} \oplus N_{i}$ are split rank 2 bundles and $L_{i}^{\prime \prime}$ are arbitrary lines bundles on $Z^{\prime}$.

We iterate the procedure by selecting a sufficiently positive divisor $Z^{\prime \prime} \subset Z^{\prime}$. Since the dimensions of the divisors are dropping, the procedure terminates when dimension 0 is reached with the elements

$$
[\underbrace{\mathbb{P}^{1} \times \cdots \times \mathbb{P}^{1}}_{n}, \underbrace{\mathcal{O}\left(l_{1}, \ldots, l_{n}\right), \ldots, \mathcal{O}\left(l_{1}, \ldots, l_{n}\right)}_{r}] \in \omega_{n, 1^{r}}(k)
$$

with $l_{i} \in\{0,1\}$. These elements are covered by Proposition 10 .

\subsection{Proof of Theorem 9}

We prove the result by induction on $n$. The $n=0$ case is clear. We assume the result for all $n^{\prime}<n$.

Using Theorem 9 for $n^{\prime}<n$, we conclude that the grade $n$ part of $\mathfrak{m} \cdot \omega_{*, 1^{r}} \otimes_{\mathbb{Z}} \mathbb{Q}$ is equal to the $\mathbb{Q}$-linear span of

$$
\left\{\phi\left(\lambda,\left(m_{1}, \ldots, m_{r}\right)\right) \mid\left(\lambda,\left(m_{1}, \ldots, m_{r}\right)\right) \in \mathcal{P}_{n, 1^{r}}, \sum_{i=1}^{r} m_{i}<n\right\}
$$


in $\omega_{n, 1^{r}}(k) \otimes_{\mathbb{Z}} \mathbb{Q}$. By Proposition 11 , we see

$$
\operatorname{dim}\left(\omega_{n, 1^{r}}(k) \otimes_{\mathbb{Z}} \mathbb{Q}\right) \leq\left|\mathcal{P}_{n, 1^{r}}\right| .
$$

Since we have already established the reverse inequality in Section 2.3, we obtain

$$
\operatorname{dim}\left(\omega_{n, 1^{r}}(k) \otimes_{\mathbb{Z}} \mathbb{Q}\right)=\left|\mathcal{P}_{n, 1^{r}}\right|,
$$

concluding the proof of Theorem 9.

\section{Higher rank}

\subsection{Splitting}

As before, let $\mathfrak{m} \subset \omega_{*}(k)$ be the ideal generated by all elements of positive dimension. Since $\omega_{*, r}(k)$ is an $\omega_{*}(k)$-module, we can define the graded quotient

$$
\tilde{\omega}_{*, r}(k)=\omega_{*, r}(k) / \mathfrak{m} \cdot \omega_{*, r}(k), \quad \widetilde{\omega}_{*, r}(k)=\bigoplus_{n=0}^{\infty} \widetilde{\omega}_{n, r}(k) .
$$

For $(\lambda, \mu) \in \mathcal{P}_{n, r}$, let $\widetilde{\phi}(\lambda, \mu) \in \widetilde{\omega}_{n, r}(k)$ denote the class of $\phi(\lambda, \mu)$ in the quotient.

Proposition 12. Let $Y \in \mathbf{S m}_{k}$ be a projective variety of dimension $n$ with rank $r$ vector bundle $E$. Then $[Y, E] \in \widetilde{\omega}_{n, r}(k)$ lies in the $\mathbb{Z}$-linear span of

$$
\left\{\tilde{\phi}(\lambda, \mu)\left|(\lambda, \mu) \in \mathcal{P}_{n, r},\right| \mu \mid=n\right\}
$$

in $\widetilde{\omega}_{n, r}(k)$.

For the proof of Proposition 12, we will require the following basic result.

Lemma 13. There exists a nonsingular projective variety $\widehat{Y}$ and a birational morphism $\widehat{Y} \rightarrow Y$ for which the pull-back of $E$ to $\widehat{Y}$ has a filtration by subbundles

$$
0=E_{0} \subset E_{1} \subset \cdots \subset E_{r}=E
$$

satisfying $\operatorname{rank}\left(E_{i} / E_{i-1}\right)=1$.

Proof. Consider the complete flag variety over $Y, \pi: \mathbb{F}(E) \rightarrow Y$. There is a rational section $s$ of $\pi$. The variety $\widehat{Y}$ is obtained from the resolution of singularities of the graph closure of $s$ in $Y \times \mathbb{F}(E)$.

To prove Proposition 12 , let $[Y, E]$ be given. Since

$$
[\widehat{Y} \rightarrow Y]=[Y \rightarrow Y] \in \widetilde{\omega}_{n}(Y)
$$

by (2.1), we conclude

$$
[\widehat{Y} \rightarrow Y, E]=[Y \rightarrow Y, E] \in \widetilde{\omega}_{n, r}(Y)
$$

as before. After pushing forward to $\operatorname{Spec}(k)$, we obtain

$$
[\widehat{Y}, E]=[Y, E] \in \widetilde{\omega}_{n, r}(k) .
$$


On $\widehat{Y}$, let $L_{1}, \ldots, L_{r}$ be the list of line bundle obtained from the subquotients of the filtration of $E$. Sending the extension parameters to 0 , we see

$$
[\widehat{Y}, E]=\left[\widehat{Y}, L_{1} \oplus \cdots \oplus L_{r}\right] \in \omega_{n, r}(k) .
$$

Finally, Proposition 11 applied to the list $\left[\widehat{Y}, L_{1}, \ldots, L_{r}\right]$ concludes the proof of Proposition 12.

\subsection{Proofs of Theorems 1 and 4}

We prove the result by induction on $n$. The $n=0$ case is clear. We assume the result for all $n^{\prime}<n$.

Using Theorem 1 for $n^{\prime}<n$, we conclude that the grade $n$ part of $\mathfrak{m} \cdot \omega_{*, r} \otimes_{\mathbb{Z}} \mathbb{Q}$ is equal to the $\mathbb{Q}$-linear span of

$$
\left\{\widetilde{\phi}(\lambda, \mu)\left|(\lambda, \mu) \in \mathcal{P}_{n, r},\right| \mu \mid<n\right\}
$$

in $\omega_{n, r}(k) \otimes_{\mathbb{Z}} \mathbb{Q}$. By Proposition 12 , we see

$$
\operatorname{dim}\left(\omega_{n, r}(k) \otimes_{\mathbb{Z}} \mathbb{Q}\right) \leq\left|\mathcal{P}_{n, r}\right| .
$$

Since we have already established the reverse inequality in Section 1.2.3, we obtain

$$
\operatorname{dim}\left(\omega_{n, r}(k) \otimes_{\mathbb{Z}} \mathbb{Q}\right)=\left|\mathcal{P}_{n, r}\right|,
$$

concluding the proof of Theorems 1 and 4 .

\subsection{Proof of Theorem 2}

Since Proposition 12 holds over $\mathbb{Z}$, we see $\omega_{n, r}(k)$ is generated over $\mathbb{Z}$ by

$$
\left\{\phi(\lambda, \mu)\left|(\lambda, \mu) \in \mathcal{P}_{n, r},\right| \mu \mid=n\right\}
$$

and the subgroups

$$
\omega_{n}(k) \cdot \omega_{0, r}(k), \quad \omega_{n-1}(k) \cdot \omega_{1, r}(k), \ldots, \omega_{1}(k) \cdot \omega_{n-1, r}(k) .
$$

We now prove Theorem 2 by induction on $n$. Certainly, $\omega_{i}(k)$ is a free $\mathbb{Z}$-module of rank equal to the number of partitions of $i$. Using the induction hypothesis, we see $\omega_{n, r}(k)$ has $\left|\mathcal{P}_{n, r}\right|$ generators over $\mathbb{Z}$. Since we know $\operatorname{dim}\left(\omega_{n, r}(k) \otimes_{\mathbb{Z}} \mathbb{Q}\right)=\left|\mathcal{P}_{n, r}\right|$, no relations among these generators are possible.

\subsection{Product structure}

There is a natural commutative ring structure on

$$
\omega_{*,+}(k)=\bigoplus_{r=1}^{\infty} \omega_{*, r}(k)=\bigoplus_{n=0}^{\infty} \bigoplus_{r=1}^{\infty} \omega_{n, r}(k)
$$

given by external product

$$
\left[Y_{1}, E_{1}\right] \cdot\left[Y_{2}, E_{2}\right]=\left[Y_{1} \times Y_{2}, p_{1}^{*}\left(E_{1}\right) \otimes p_{2}^{*}\left(E_{2}\right)\right] .
$$


Here, $p_{1}$ and $p_{2}$ are the projections of $Y_{1} \times Y_{2}$ onto the first and second factors respectively. There is an inclusion of rings

$$
\omega_{*}(k) \hookrightarrow \omega_{*,+}(k), \quad[Y] \mapsto[Y, \mathcal{O}] .
$$

By the basis result of Theorem 1 , the product on $\omega_{*,+}(k) \otimes_{\mathbb{Z}} \mathbb{Q}$ is completely determined by the special case

$$
\left[\mathbb{P}^{a}, \mathcal{O}(1)\right] \cdot\left[\mathbb{P}^{b}, \mathcal{O}(1)\right]=\left[\mathbb{P}^{a} \times \mathbb{P}^{b}, \mathcal{O}(1,1)\right] .
$$

Question. What is the decomposition of $\left[\mathbb{P}^{a} \times \mathbb{P}^{b}, \mathcal{O}(1,1)\right]$ in the basis of $\omega_{a+b, 1}(k) \otimes_{\mathbb{Z}} \mathbb{Q}$ given in Theorem 1 ?

Of course, Theorem 4 provides a computational approach to the question for any fixed $a$ and $b$. Is there a closed formula or any structure in the answer?

\section{Results over $X$}

\subsection{Surjectivity}

Following the notation of Section 2.4, let

$$
\widetilde{\omega}_{*, 1^{r}}(X)=\omega_{*, 1^{r}}(X) / \mathfrak{m} \cdot \omega_{*, 1^{r}}(X), \quad \widetilde{\omega}_{*, 1^{r}}(X)=\bigoplus_{n=0}^{\infty} \widetilde{\omega}_{n, 1^{r}}(X) .
$$

Consider the element

$$
\left[Y \rightarrow X, L_{1}, \ldots, L_{r}\right] \in \omega_{n, 1^{r}}(X) .
$$

If all the $L_{i}$ are globally generated on $Y$, then there exists a projective morphism

$$
f: Y \rightarrow X \times \mathbb{P}^{d_{1}} \times \cdots \times \mathbb{P}^{d_{r}}, \quad L_{i}=f^{*}\left(\mathcal{O}_{\mathbb{P}^{d_{i}}}(1)\right) .
$$

We view $f$ as determining an element of algebraic cobordism,

$$
\left[f: Y \rightarrow X \times \mathbb{P}^{d_{1}} \times \cdots \times \mathbb{P}^{d_{r}}\right] \in \omega_{n}\left(X \times \mathbb{P}^{d_{1}} \times \cdots \times \mathbb{P}^{d_{r}}\right) .
$$

The Chow group $A_{n}\left(X \times \mathbb{P}^{d_{1}} \times \cdots \times \mathbb{P}^{d_{r}}\right)$ is generated over $A_{*}(X)$ by linear subvarieties

$$
\iota_{m_{1}, \ldots, m_{r}}: \mathbb{P}^{m_{1}} \times \cdots \times \mathbb{P}^{m_{r}} \hookrightarrow \mathbb{P}^{d_{1}} \times \cdots \times \mathbb{P}^{d_{r}}
$$

where $\sum_{i=1}^{r} m_{i} \leq n$. Using (2.1) for $X \times \mathbb{P}^{d_{1}} \times \cdots \times \mathbb{P}^{d_{r}}$, we see $[f]$ is a $\mathbb{Z}$-linear combination of elements of the form

$$
\left[\iota \times \iota_{m_{1}, \ldots, m_{r}}\right] \in \widetilde{\omega}_{n}\left(X \times \mathbb{P}^{d_{1}} \times \cdots \times \mathbb{P}^{d_{r}}\right)
$$

where $\iota: W \rightarrow X$ is a resolution of singularities of an irreducible subvariety of $X$ and

$$
n=\operatorname{dim}(W)+\sum_{i=1}^{r} m_{i} .
$$


Concluding as in the proof of Proposition 10, we find that

$$
\left[Y \rightarrow X, L_{1}, \ldots, L_{r}\right] \in \widetilde{\omega}_{n, 1^{r}}(X)
$$

lies in the subspace spanned by products of elements of $\omega_{\delta}(X)$ with basis terms of $\omega_{n-\delta, 1^{r}}(k)$.

The projective bundle analysis in the proof of Proposition 11 occurs entirely over $Y$ and thus over $X$. Hence, we can remove the global generation hypothesis on the bundles $L_{i}$ just as before.

Since the splitting of Lemma 13 also occurs over $Y$, we conclude that the composition

$$
\omega_{*}(X) \otimes_{\omega_{*}(k)} \omega_{*, r}(k) \stackrel{\gamma_{X}}{\longrightarrow} \omega_{*, r}(X) \rightarrow \widetilde{\omega}_{*, r}(X)
$$

is surjective.

Proposition 14. The natural map

$$
\gamma_{X}: \omega_{*}(X) \otimes_{\omega_{*}(k)} \omega_{*, r}(k) \rightarrow \omega_{*, r}(X)
$$

is surjective.

Proof. We have already seen $\gamma_{X}$ surjects onto $\omega_{*, r}(X) / \mathfrak{m} \cdot \omega_{*, r}(X)$. But then

$$
\mathfrak{m} \cdot \omega_{*}(X) \otimes_{\omega_{*}(k)} \omega_{*, r}(k)
$$

surjects via $\gamma_{X}$ onto $\mathfrak{m} \cdot \omega_{*, r}(X) / \mathfrak{m}^{2} \cdot \omega_{*, r}(X)$. The result follows by iteration since $\bigcap_{i \geq 1} \mathfrak{m}^{i}=0$.

\subsection{Injectivity}

Let $c_{1}, \ldots, c_{r}$ be variables with $c_{i}$ of degree $i$. Let $\Psi$ be the space of polynomials in $c_{1}, \ldots, c_{r}$ with $\mathbb{Z}$ coefficients. For homogeneous $\psi \in \Psi$ of degree $d$, there are natural Chern operations

$$
C_{\psi}: \omega_{*, r}(X) \rightarrow \omega_{*-d}(X)
$$

defined by

$$
C_{\psi}([Y \stackrel{f}{\rightarrow} X, E])=f_{*}\left(\psi\left(c_{1}(E), \ldots, c_{r}(E)\right) \cap[Y \rightarrow Y]\right) \in \omega_{*-d}(X)
$$

where the action of $\psi$ on the right is via the standard Chern class operations $[6$, Section 7.4] in algebraic cobordism.

To show definition (4.1) respects the double point relation in $\omega_{*, r}(X)$, we argue as follows. Suppose $g: Y \rightarrow X \times \mathbb{P}^{1}$ is a projective morphism for which the composition $\pi=p_{2} \circ g: Y \rightarrow \mathbb{P}^{1}$ is a double point degeneration over $0 \in \mathbb{P}^{1}$, and $E$ is a rank $r$ vector bundle on $Y$. The Chern operation $\psi\left(c_{1}(E), \ldots, c_{r}(E)\right)$ is well-defined on $\omega_{*}(Y)$,

$$
\psi: \omega_{*}(Y) \rightarrow \omega_{*-d}(Y)
$$


Hence, for regular values $\zeta \in \mathbb{P}^{1}(k)$ of $\pi$,

$$
\psi \cap\left(\left[Y_{\zeta} \rightarrow Y\right]-[A \rightarrow Y]-[B \rightarrow Y]+[\mathbb{P}(\pi) \rightarrow Y]\right)=0 \quad \text { in } \omega_{*}(Y) .
$$

Pushing forward to $X$ and using the functoriality of the Chern class, we obtain

$$
\begin{aligned}
C_{\psi}\left(\left[Y_{\zeta} \rightarrow X, E_{Y_{\zeta}}\right]\right)-C_{\psi}\left(\left[A \rightarrow X, E_{A}\right]\right) & \\
& \quad-C_{\psi}\left(\left[B \rightarrow X, E_{B}\right]\right)+C_{\psi}\left(\left[\mathbb{P}(\pi) \rightarrow X, E_{\mathbb{P}(\pi)}\right]\right)=0 \quad \text { in } \omega_{*}(X),
\end{aligned}
$$

which is the required compatibility.

By the characterization of $\omega_{*, r}(k)$ in Theorem 2, we have

$$
\omega_{*}(X) \otimes_{\omega_{*}(k)} \omega_{*, r}(k)=\bigoplus_{\lambda} \omega_{*}(X) \otimes \phi(\lambda, \lambda)
$$

where the sum is over all partitions $\lambda$ of length at most $r$. Consider the pairing

$$
\rho^{X}: \omega_{*}(X) \otimes_{\omega_{*}(k)} \omega_{*, r}(k) \times \Psi \rightarrow \omega_{*}(X) \text { defined by } \rho^{X}(\zeta, \psi)=C_{\psi}\left(\gamma_{X}(\zeta)\right) .
$$

Using the basis (4.2), we see the pairing $\rho^{X}$ is triangular with 1 's on the diagonal by calculation (1.4). We have proven the following result.

Proposition 15. The natural map

$$
\gamma_{X}: \omega_{*}(X) \otimes_{\omega_{*}(k)} \omega_{*, r}(k) \rightarrow \omega_{*, r}(X)
$$

is injective.

Propositions 14 and 15 together complete the proof of Theorem 3. In fact, the proof of Theorem 3 is just a slight abstraction of the original proof of Theorem 1 .

Acknowledgments. We thank D. Abramovich, J. Li, D. Maulik, B. Totaro, and Y. Tzeng for discussions about algebraic cobordism and double point degenerations. The basis of Theorem 1 was guessed while writing [7]. Conversations with $\mathrm{M}$. Levine played an essential role. He suggested the possibility of the extension of scalars result established in Theorem 3.

Y.-P. L. was supported by NSF grant DMS-0901098. R. P. was supported by NSF grant DMS0500187.

\section{References}

[1] Bryan, J., Pandharipande, R.: Local Gromov-Witten theory of curves. J. Amer. Math. Soc. 21, 101-136 (2008) Zbl 1126.14062 MR 2350052

[2] Deshpande, D.: Algebraic cobordism of classifying spaces. arXiv:0907.4437

[3] Göttsche, L.: A conjectural generating function for numbers of curves on surfaces. Comm. Math. Phys. 196, 523-533 (1998) Zbl 0934.14038 MR 1645204

[4] Lazard, M.: Sur les groupes de Lie formels à un paramètre. Bull. Soc. Math. France 83, 251274 (1955) Zbl 0068.25703 MR 0073925

[5] Lee, Y.-P., Lin, H.-W., Wang, C.-L.: in preparation 
[6] Levine, M., Morel, F.: Algebraic Cobordism. Springer Monogr. Math., Springer, Berlin (2007) Zbl 1188.14015 MR 2286826

[7] Levine, M., Pandharipande, R.: Algebraic cobordism revisited. Invent. Math. 176, 63-130 (2009) Zbl 1210.14025 MR 2485880

[8] Heller, J., Malagon-Lopez, J.: in preparation

[9] Maulik, D., Nekrasov, N., Okounkov, A., Pandharipande, R.: Gromov-Witten theory and Donaldson-Thomas theory I. Compos. Math. 142, 1263-1285 (2006) Zbl 1108.14046 MR 2264664

[10] Maulik, D., Nekrasov, N., Okounkov, A., Pandharipande, R.: Gromov-Witten theory and Donaldson-Thomas theory II. Compos. Math. 142, 1286-1304 (2006) Zbl 1108.14047 MR 2264665

[11] Stong, R.: Notes on Cobordism Theory. Princeton Univ. Press, Princeton, NJ (1968) Zbl 0181.26604 MR 0248858

[12] Totaro, B.: The Chow ring of a classifying space. In: Algebraic $K$-theory (Seattle, 1997), Proc. Sympos. Pure Math. 67, Amer. Math. Soc., 249-281 (1999) Zbl 0967.14005 MR 1743244

[13] Tzeng, Y.: Stanford thesis (2010)

[14] Yagita, N.: Applications of Atiyah-Hirzebruch spectral sequences for motivic cobordism. Proc. London Math. Soc. 90, 783-816 (2005) Zbl 1086.55005 MR 2137831 